\title{
MicroRNAs play a role in chondrogenesis and osteoarthritis (Review)
}

\author{
CHUANLONG WU*, BO TIAN*, XINHUA QU*, FENGXIANG LIU, TINGTING TANG, \\ AN QIN, ZHENAN ZHU and KERONG DAI
}

\begin{abstract}
Shanghai Key Laboratory of Orthopaedic Implants, Department of Orthopaedics, Shanghai Ninth People's Hospital, Shanghai Jiaotong University School of Medicine, Shanghai 200011, P.R. China
\end{abstract}

Received November 25, 2013; Accepted April 8, 2014

DOI: $10.3892 / \mathrm{ijmm} .2014 .1743$

\begin{abstract}
Osteoarthritis (OA) is one of the most widespread degenerative joint diseases affecting the elderly. Research into the regulatory mechanisms underlying the pathogenesis of OA is therefore warranted, and over the past decade, there has been an increased focus on the functional role of microRNAs (miRNAs or miRs). In this systematic review, we aimed to review the evidence implicating miRNAs in the pathogenesis of chondrogenesis and OA. Systematic reviews of PubMed and Embase were performed to search for studies using strings of miRNAs, non-coding RNAs, cartilage, chondrocytes, chondrogenesis, chondrocytogenesis and OA. The identified studies were retrieved, and the references provided were searched. The selected studies were required to focus on the role of miRNAs in chondrogenesis and OA. The results of this review indicated that more than 25 miRNAs have been implicated in chondrogenesis and OA. In particular, chondrocytogenesis, chondrogenic differentiation, chondrocyte proliferation, chondrocyte hypertrophy, endochondral ossification, and proteolytic enzyme regulation are targeted or facilitated by more than 1 miRNA. To date, limited efforts have been performed to evaluate translational applications for this knowledge. Novel therapeutic strategies have been developed and are under investigation to selectively modulate miRNAs, which could potentially enable personalized OA therapy. miRNAs appear to be important modulators of chondrogenesis and OA. Their expression is frequently altered in OA, and many are functionally implicated in the pathogenesis of the disease. The translational roles and therapeutic potential of miRNAs remains to be evaluated.
\end{abstract}

Correspondence to: Dr An Qin or Dr Zhenan Zhu, Department of Orthopaedics, Shanghai Ninth People's Hospital, Shanghai Jiaotong University School of Medicine, 639 Zhizaoju Road, Shanghai 200011, P.R. China

E-mail: dr.qinan@gmail.com

E-mail: zhenan_zhu@126.com

*Contributed equally

Key words: microRNA, chondrogenesis, chondrocytes, osteoarthritis

\section{Contents}

1. Introduction

2. Literature search and literature selection

3. miRNAs in chondrocytogenesis

4. miRNAs in osteoarthritis

5. Conclusion

\section{Introduction}

Osteoarthritis (OA) is a highly prevalent degenerative joint disease characterized by the progressive destruction of articular cartilage, synovial hyperplasia and sclerosis of the subchondral bone (1-4). In the United States, knee and/or hip $\mathrm{OA}$ are the most common causes of walking-related disability among older adults (5). With the increasing incidence of obesity and an aging population, the public health consequences of OA and OA-related disability are have become a pressing issue, and novel therapeutic strategies are urgently required (6).

Cartilage is classified histologically into hyaline, elastic, and fibrocartilaginous, depending on the molecular composition (7). Articular cartilage is a form of hyaline cartilage and is divided into 4 zones: superficial, transitional, radial and calcified cartilage (8). These zones are characterized by a distinct organization of the collagen network, and by differences in the amounts and types of proteoglycans. In healthy articular cartilage, the principal molecular component is type II collagen, although collagens III, VI, IX, X, XI, XII and XIV all exist in the mature matrix (9-11). Additionally, aggrecan, a large chondroitin sulfate proteoglycan, is the main proteoglycan present in cartilage, which also includes syndecans, glypican, decorin, biglycan, fibromodulin, lumican, epiphycan and perlecan (12). Apart from the extracellular matrix (ECM), chondrocytes are the only cells found in healthy cartilage. Chondrocytes are arranged in a zonal stratification and embedded in the arcade-like network of collagen fibrils intermingled with proteoglycans (11). Chondrocytes respond to their microenvironment to regulate articular cartilage homeostasis by balancing the synthesis and degradation of ECM components. The ECM is degraded by proteolytic enzymes, including matrix metalloproteinases (MMPs) and the 'A disintegrinand metalloproteinase domain with thrombospondin-like motifs' 
family (ADAMTS) (13-15). In OA, cartilage degradation occurs in 2 phases: the degradative and biosynthetic phase. In the degradative phase, ADAMTS and MMPs digest the ECM in the presence of inflammatory cytokines. Consequently, in the biosynthetic phase, the chondrocytes attempt to repair the damaged ECM. However, matrix synthesis is likewise inhibited by inflammatory factors through the downregulation of ECM genes, thus accelerating cartilage erosion (14).

microRNAs (miRNAs or miRs) are a class of non-coding single-stranded RNAs of 18-22 nucleotides (nt). They regulate gene expression at the post-transcriptional, level and therefore affect cell fate during cell proliferation and differentiation (16). This regulatory mechanism is often modulated by the promotion of mRNA degradation and/or the repression of translation through sequence-specific interactions with the 3'-untranslated regions (3'-UTRs) of specific mRNA targets. Genes encoding miRNAs are transcribed by RNA polymerase II or III to generate Pri-miRNAs, which are processed to Pre-miRNAs by Drosha in the nucleus. Pre-miRNAs are exported to the cytoplasm where they are further processed by the RNase III ribonuclease, Dicer, to generate 22 -nt singlestranded mature miRNAs (17-21), which then combine with the RNA-induced silencing complex (RISC) by the core unit Argonaute (Ago) $(16,22,23)$. The miRNA-RISC complex binds target mRNAs and mediates mRNA translational repression or degradation (24-27) (Fig. 1).

miRNAs are evolutionarily conserved and have been found in various organisms (28). In the human musculoskeletal system, the conditional knockout of Dicer in the limb mesenchyme during the early stages of mice embryonic development leads to the formation of smaller limbs (29), suggesting that miRNAs play a prominent role in skeletal development. Furthermore, Dicer-null growth plates display a lack of chondrocyte proliferation but an enhanced hypertrophic chondrocyte differentiation, indicating that Dicer and miRNAs exhibit distinct functional effects at different stages of chondrocyte development (29). Given the importance of miRNAs in chondrocyte development, we reviewed recent research on miRNA regulation in cartilage biology, with the aim to further understand chondrogenesis and OA development, and the development of potential gene therapeutic strategies for OA prevention and treatment.

\section{Literature search and literature selection}

Literature search. A systematic literature review was conducted using the PubMed and Embase databases on April 1, 2013. Several combinations of terms and expressions were evaluated, including both MeSH and free text terms. By analyzing the articles retrieved from each combination, we selected the following final search expression: (cartilage OR chondrocyte, chondrogenesis OR chondrocytogenesis OR osteoarthritis) and (microRNA OR non-coding RNA). Additionally, we manually searched the reference lists of the identified aritcles. We used the Preferred Reporting Items of Systematic Reviews and Meta-Analyses (PRISMA) guidelines in the planning and execution of this study.

Literature selection. We selected articles, written in the English language, in which scientific detail and reporting were sufficient to enable our understanding of the material, and which presented novel findings. In cases of multiple or serial reports, we selected either the first or the most detailed report for inclusion. We preferentially selected articles in which mechanistic data supported observational findings.

Our literature search retrieved 457 studies. We selected 59 studies which were of sufficient reporting rigor or novelty, and provided mechanistic data of the biology of chondrogenesis and $\mathrm{OA}$.

\section{3. miRNAs in chondrocytogenesis}

Chondrocytes are derived from the chondrogenic differentiation of mesenchymal stem cells (MSCs) (30). The process of chondrogenic differentiation mainly includes 6 phases: mesenchymal cells (chondroprogenitors), condensed mesenchymal cells, chondrocytes, proliferating chondrocytes, pre-hypertrophic chondrocytes and hypertrophic chondrocytes (31). A number of transcription factors and cytokines influence discrete steps in the chondrocyte differentiation pathway. These include members of the Sox family [Sox9, Sox 5 and Sox6 $(32,33)]$, bone morphogenetic proteins (BMPs) (34), connective tissue growth factor/cysteine-rich 61/nephroblastoma overexpressed (CCN) family protein 2 (CCN2) (35), as well as others. Additionally, several miRNAs participate in the regulation of chondrogenesis by targeting these transcription factors and growth cytokines (Table I and Fig. 2).

miRNAs and the Sox family in chondrocytogenesis: miR-101, $m i R-145$ and $m i R-194$. Sox proteins belong to the high-mobility group (HMG) superfamily (36). Sox genes are indispensable to multiple aspects of cartilage development by activating the expression of cartilage-specific ECM components. Sox 9 is a transcription factor belonging to the Sox protein family, which is essential for chondrogenesis, and has been designated a 'master regulator' of the chondrocyte phenotype $(37,38)$. Sox 9 is expressed in all chondroprogenitor cells, predominantly in mesenchymal condensations and cartilage (39). Sox 9 can bind to and activate chondrocyte-specific enhancer elements in Col2a1, Col9a1, Col11a2 and aggrecan to induce cartilage-specific gene expression (40-43). Additionally, Sox 9 prevents chondrocyte hypertrophy and exerts a re-differentiation effect on dedifferentiated osteoarthritic chondrocytes $(44,45)$. Of note, miR-101 participates in the interleukin (IL(-1 $\beta$-induced downregulation of collagen type II and aggrecan, possibly functioning through its target gene, Sox9 (46). Therefore, miR-101 inhibition is effective in preventing IL-1 $\beta$-induced chondrocyte ECM degradation (46). Additionally, miR-145 expression is gradually reduced during transforming growth factor- $\beta$ (TGF- $\beta$ )-induced chondrogenic differentiation. Through the 3'-UTR-reporter assay and gain- or loss-of-function experiments, Sox 9 has also identified as an miR-145 target (32). This was further supported by the fact that $\operatorname{Sox} 9$ was identified as a direct target of miR-145 in human chondrocytes (33). Thus, the attenuation of miR-145 expression can positively regulate $\operatorname{Sox} 9$ expression, resulting in the promotion of chondrogenic differentiation.

In addition to Sox9, Sox 5 and Sox6 (which share a high degree of sequence identity) belong to a different subgroup of Sox proteins and present no sequence homology with Sox9, apart from the HMG-box. Sox 5 and Sox6 are co-expressed with Sox 9 during chondrogenic differentiation. Both genes are 


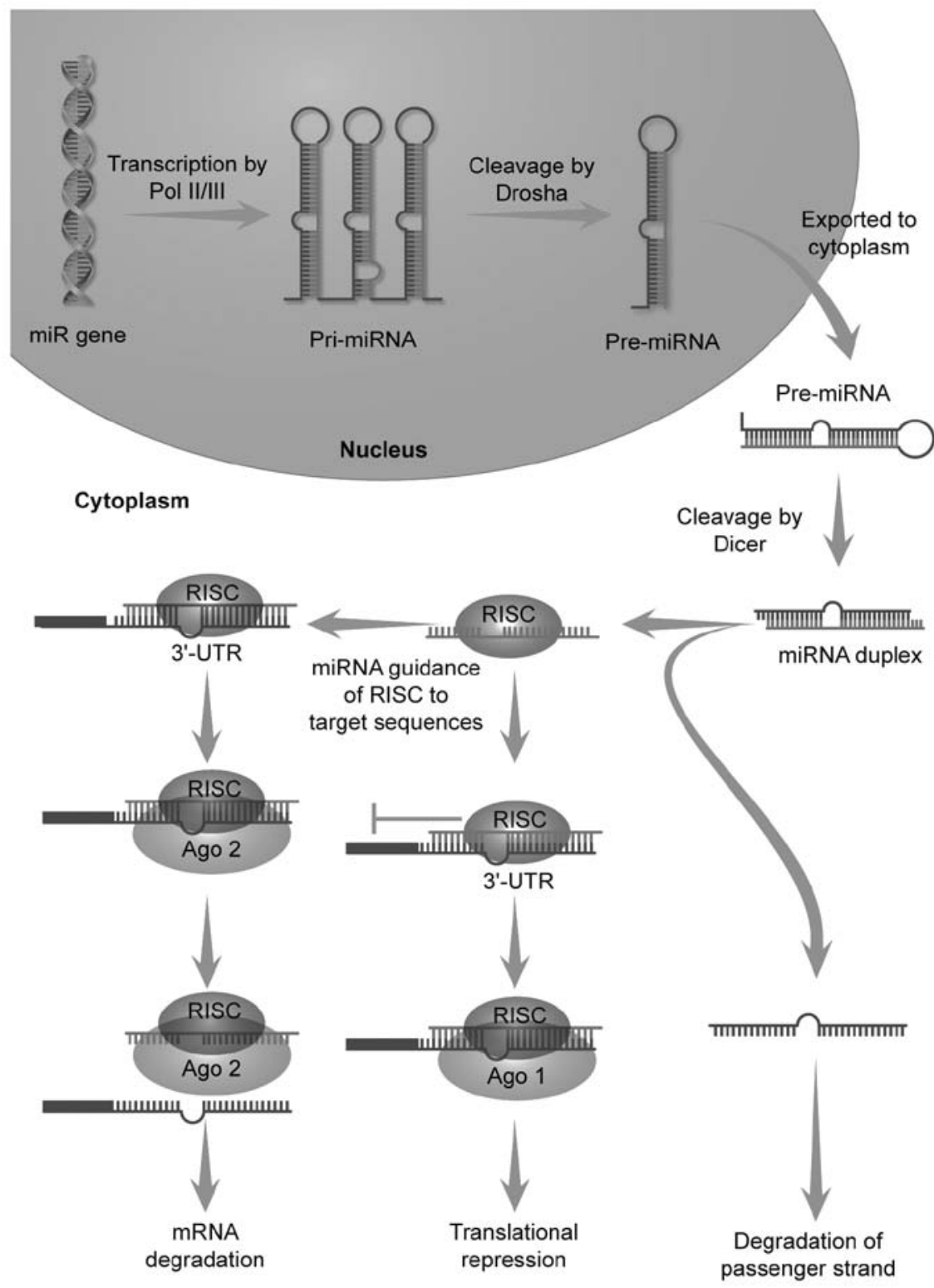

Figure 1. Biogenesis of microRNAs (miRNAs). Genes encoding miRNAs are transcribed by RNA polymerase II or III to generate Pri-miRNAs, which are processed to Pre-miRNAs by Drosha in the nucleus. Pre-miRNAs are exported to the cytoplasm where they are further processed by the RNase III ribonuclease Dicer to generate $22 \mathrm{nt}$ single-stranded mature miRNAs (17-21), which then combine with the RNA-induced silencing complex (RISC) by the core unit Argonaute (Ago) $(16,22,23)$. The miRNA-RISC complex binds target mRNAs and mediates mRNA translational repression or degradation.

also expressed in several non-chondrogenic tissues. Owing to the presence of a highly conserved coiled-coil domain, Sox 5 and Sox6 form homodimers and heterodimers, which bind much more efficiently to pairs of HMG-box-binding sites than to single binding sites. Unlike Sox9, Sox 5 and Sox6 do not contain a transcriptional activation domain. Sox 5 and Sox6 cooperate with Sox9 to activate the Col2al enhancer and expression of Col2a1 and aggrecan genes (39). Of note, Sox 5 was identified as a target of miR-194 based on luciferase assay analysis (47). The expression of miR-194 was gradually downregulated during the chondrogenic differentiation of human adipose-derived stem cells (hASCs) (47). However, miR-194 was upregulated under pathological conditions (e.g., OA). In gain- or loss-of-function experiments, the downregulation of miR-194 increased the expression of its direct target gene, Sox 5, resulting in enhanced chondrogenic differentiation (47).
Taken together, these findings suggest that miR-101, miR-145 and miR-194 are important for chondrogenesis by targeting the Sox family.

miRNAs and the BMP family in chondrocytogenesis: $m i R-199 a^{*}, m i R-140$ and $m i R-92 a$. BMPs are members of the TGF- $\beta$ family that bind to type II and I serine-threonine kinase receptors, and transduce signals through Smad and nonSmad signaling pathways (48). BMPs, particularly BMP2 and BMP4, are very powerful growth factors that induce cartilage formation by stimulating chondrocyte differentiation (36). Smad signaling is important for BMP signaling. Smad1 is a downstream signaling molecule of BMP2 (49-51). Of note, Smad1 was identified as the direct target of miR-199a*, which is specifically expressed in the skeletal system (52). miR-199a* significantly inhibited early chondrogenesis, as revealed by 
Table I. Summary of microRNAs in chondrocytes.

\begin{tabular}{|c|c|c|c|c|}
\hline microRNAs & Targets & Regulatory role & Authors/(Refs.) & Study models \\
\hline miR-101 & Sox9 & Chondrocyte differentiation (-) & Dai et al (46) & Rat chondrocytes \\
\hline miR-145 & Sox9 & Chondrocyte differentiation (-) & Yang et al (32) & Murine MSCs \\
\hline $\operatorname{miR}-199 a^{*}$ & Smad1 & Early chondrocyte differentiation (-) & Lin et al (52) & Pluripotent $\mathrm{C} 3 \mathrm{H} 10 \mathrm{~T} 1 / 2$ stem cells \\
\hline miR-92a & Noggin3 & $\begin{array}{l}\text { Proliferation and differentiation of } \\
\text { chondrogenic progenitors }(+)\end{array}$ & Ning et al (34) & Fish embryos \\
\hline $\operatorname{miR}-488$ & $\begin{array}{l}\text { Not detected } \\
\text { (TIMP?) }\end{array}$ & Condensed mesenchymal cells $(+)$ & Song et al (59) & $\begin{array}{l}\text { Mesenchymal cells derived from the } \\
\text { distal tips of Hamburger-Hamilton }(\mathrm{HH}) \\
\text { stage } 22 / 23 \text { embryo leg buds of fertilized } \\
\text { white Leghorn chicken eggs }\end{array}$ \\
\hline miR-34a & EphA5 & Condensed mesenchymal cells (-) & Kim et al (60) & $\begin{array}{l}\text { Chick limb mesenchymal cells; } \\
\text { human chondrocytes }\end{array}$ \\
\hline $\operatorname{miR}-221$ & MDM2 & Proliferation of chondroprogenitors (-) & Kim et al (60) & $\begin{array}{l}\text { Mesenchymal cells derived from the distal } \\
\text { tips of HH stage } 22 / 23 \text { embryo leg buds } \\
\text { of fertilized White Leghorn chicken eggs }\end{array}$ \\
\hline miR-194 & Sox 5 & $\begin{array}{l}\text { Differentiated chondrocyte and } \\
\text { proliferating chondrocyte (-) }\end{array}$ & Xu et al (47) & $\begin{array}{l}\text { The human ASCs; } 2 \text { weeks old Wistar } \\
\text { male rats; articular chondrocytes isolated } \\
\text { from the knee joints of juvenile rats }\end{array}$ \\
\hline \multirow[t]{6}{*}{$\operatorname{miR}-140$} & HDAC4 & Hyperdrophic chondrocyte (+) & Tuddenham et al (71) & Mouse cells \\
\hline & CXCL12 & Hyperdrophic chondrocyte (-) & Nicolas et al (72) & Mouse cells \\
\hline & SP1 & Chondrocyte proliferation (+) & Yang et al (67) & Limb bud mesenchymal cells \\
\hline & DNPEP & Endochondral bone formation (+) & Nakamura et al (58) & miR-140-null mice; primary chondrocyte \\
\hline & BMP2 & Endochondral bone formation (-) & Nicolas et al (56) & $\begin{array}{l}\text { Chicken primary chondrocytes; } \\
\text { primary human chondrocytes }\end{array}$ \\
\hline & Smad3 & Chondrocyte differentiation (-) & Pais et al (57) & Mouse cells \\
\hline miR-1 & Not detected & Hyperdrophic chondrocyte (-) & Sumiyoshi et al (68) & $\begin{array}{l}\text { Human chondrocytic HCS- } 2 / 8 \text { cells; } \\
\text { primary chicken chondrocytes }\end{array}$ \\
\hline $\operatorname{miR}-365$ & HDAC4 & Hyperdrophic chondrocyte (+) & Guan et al (69) & Chicken primary proliferative chondrocytes \\
\hline miR-18a & CCN2/CTGF & Endochondral bone formation (-) & Ohgawara et al (35) & $\begin{array}{l}\text { Human chondrosarcoma-derived } \\
\text { chondrocytic cell line, HCS- } 2 / 8 \text {; } \\
\text { human cervical cancer-derived cell line, } \\
\text { HeLa; chicken sternum chondrocytes }\end{array}$ \\
\hline $\operatorname{miR}-337$ & TGFBR2 & Endochondral bone formation (-) & Zhong et al (73) & $\begin{array}{l}\text { Femoral head cartilage tissues were } \\
\text { obtained from Sprague-Dawley (SD) } \\
\text { or Dark Agouti (DA) rats }\end{array}$ \\
\hline $\operatorname{miR}-23 b$ & $\begin{array}{l}\text { Protein kinase } \mathrm{A} \\
\text { (PKA) }\end{array}$ & Chondrogenic differentiation (+) & Ham et al (74) & $\begin{array}{l}\text { Human mesenchymal stem cells (hMSCs); } \\
\text { normal cell line Chinese } \\
\text { hamsterovary }(\mathrm{CHO}) \text { cells }\end{array}$ \\
\hline
\end{tabular}

TIMP, tissue inhibitor of metalloproteinases; HDAC4, histone deacetylase 4; CCN, connective tissue growth factor/cysteine-rich $61 /$ nephroblastoma overexpressed; CTGF, connective tissue growth factor; TGFBR2, transforming growth factor- $\beta$ type II receptor 2.

the reduced expression of early chondrogenesis marker genes, such as cartilage oligomeric matrix protein (COMP), type II collagen and Sox9, whereas anti-miR-199a* increased the expression of these genes. Therefore, miR-199a* acts as a negative regulator of early chondrogenic differentiation through the suppression of Smad1 in the BMP signaling pathway.

In addition to miR-199a*, miR-140 participates in the regulation of BMP signaling during cartilage development (54). miR-140 is highly expressed in chondrocytes, and miR-140 expression increases during chondrogenic differentiation from
MSCs (55). Standard luciferase assays have confirmed that BMP2 is a direct target of miR-140 (56). In addition to BMP2, Smad3 was identified as a direct target of miR-140, and the suppression of Smad3 by miR-140 can subsequently inhibit the TGF- $\beta$ pathway in chondrocytes (57). Furthermore, miR140-null mice exhibit skeletal defects, possibly due to a reduction in basal BMP signaling in miR-140-null chondrocytes (58).

miR-92a has also been identified in the regulation of the BMP signaling pathway. miR-92a is highly enriched in chondrogenic progenitors. The inactivation of miR-92a has been shown to result 


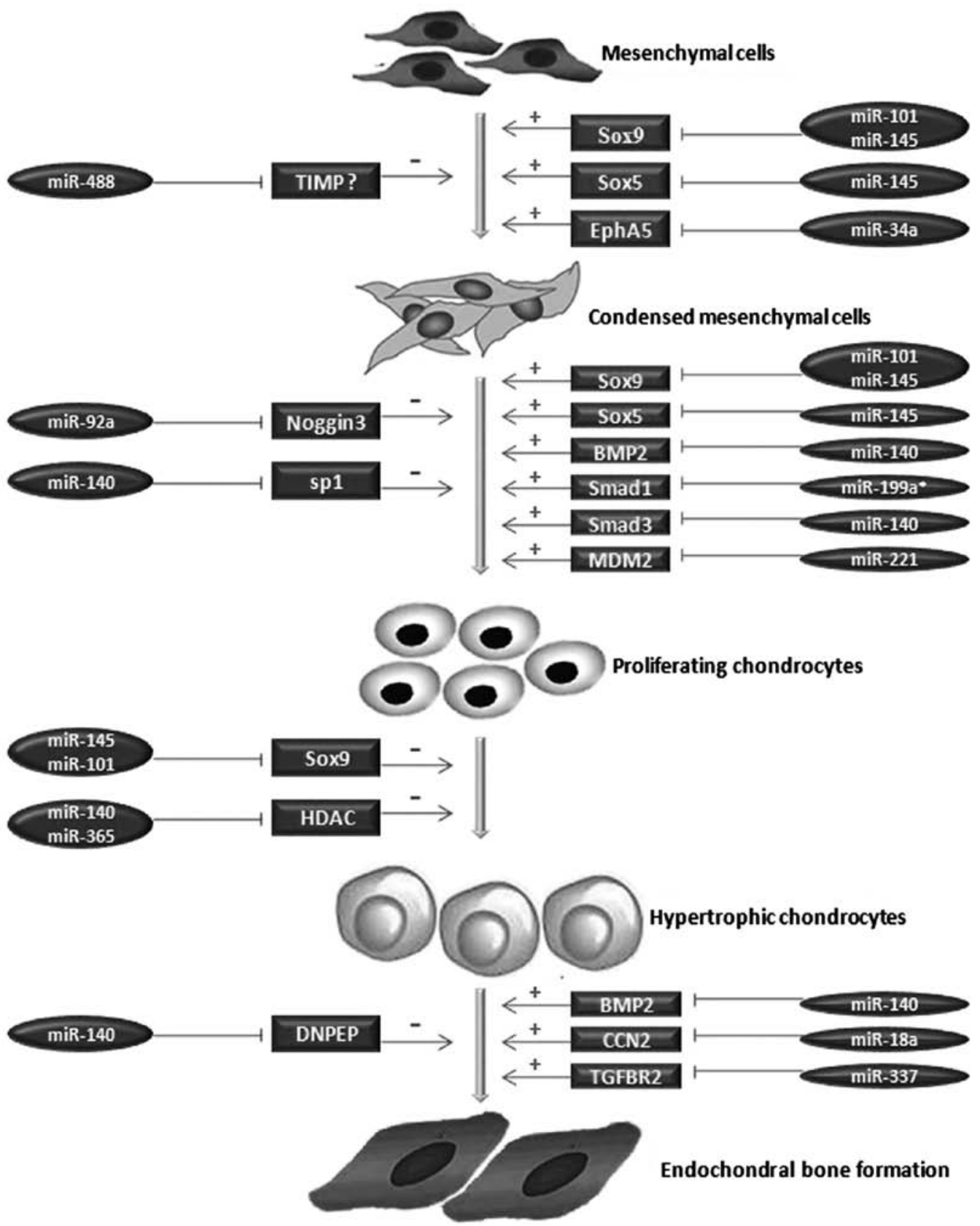

Figure 2. microRNAs (miRNAs) and their target factors in the process of chondrogenic differentiation. The roles of inhabitation are indicated by the '-' symbol and the roles of promotion are indicated by the ' + ' symbol.

in poor proliferation, impaired differentiation and the unsustainable survival of chondrogenic progenitors (34). The BMP antagonist gene, Noggin3 (Nog3), has been reported to be a direct target of miR-92a. Nog3 represses BMP activity to prevent apoptosis. Therefore, miR-92a inactivation stabilizes Nog3 mRNA and represses BMP signaling, resulting in abnormal behaviors of chondrogenic progenitors. By contrast, the ectopic expression of the miR-92a duplex reduces Nog3 mRNA levels, and thereby suppresses BMP signaling and promotes cell apoptosis. Therefore, miR-92a maintains BMP activity during cartilage formation by targeting Nog3 (34). Collectively, these data demonstrate that miR-199a*, miR-140 and miR-92a are critical for chondrogenesis by regulating the BMP signaling pathway.
miRNAs in the process of chondrogenic differentiation

miRNAs in condensed mesenchymal cells: $m i R-488$ and $m i R-34 a$. In addition to their involvement in the aforementioned 2 key families, miRNAs regulate chondrocyte differentiation at different stages. Pre-cartilage mesenchymal condensation is characterized by the production of sulfated proteoglycans and the switch from type I to II collagen synthesis. Thereafter, differentiated chondrocytes proliferate and secrete increasing amounts of ECM macromolecules until each single cell is completely surrounded by a matrix. In condensed MSCs, miR-488 expression is upregulated at the pre-condensation stage and then downregulated at the post-condensation stage. The blocking of miR-488 via antisense oligonucleotides reduces 
integrin b1 and phosphorylated focal adhesion kinase (FAK) levels, and suppresses cell motility and migration (59). In parallel with these observations, treatment with anti-miR-488 oligonucleotides has been shown to upregulate MMP-2 activity and inhibit cellular condensation (59). The direct target of miR-488 in the modulation of focal adhesion activity remains unidentified, although a possible direct target is tissue inhibitor of metalloproteinases (TIMP)-1. In summary, miR-488 is a regulator in cell-ECM interaction through the modulation of focal adhesion activity by MMP-2 during chondrogenesis of limb mesenchymal cells (59).

Additionally, miR-34a expression is induced by c-Jun $\mathrm{N}$-terminal kinase (JNK) in the chondrogenic differentiation of chick limb mesenchymal cells (60) and by IL-1 $\beta$ stimulation in rat primary chondrocytes (61). miR-34a is a negative modulator of chondrogenesis, particularly in the migration of chondroblasts, by targeting EphA5, which inhibits cellular condensation (60). Furthermore, locked nucleotide analogue (LNA)-modified miR-34a antisense can prevent the downregulation of Col2al and the upregulation of iNOS in chondrocytes (61). Therefore, miR-488 and miR-34a regulate condensed mesenchymal cells.

miRNAs in proliferating chondrocytes: miR-221 and $m i R-140$. Chondrocyte proliferation is the process for acquiring a chondrocyte phenotype, leading to the formation of unique tissues as cartilage (31). Of note, treatment with a JNK pathway inhibitor has been shwon to upregulate miR-221 expression in chondroprogenitor cells, leading to reduced proliferation and pre-cartilage condensation (62). Mouse double-minute 2 homolog (MDM2) is a relevant miR-221 target. miR-221 has been found to be both necessary and sufficient for the downregulation of MDM2 expression, which prevents slug protein degradation, and thus negatively regulates chondroprogenitor proliferation (62). Additionally, in articular cartilage, miR-221 has been suggested to be a potential regulator of the mechanotransduction pathway (63). Sp1 has been previously reported to be expressed in chondrocytes to activate ColII expression $(29,64)$ and is also a critical transcription factor in cell cycle inhibition through the activation of $\mathrm{p} 15^{\mathrm{INK} 4 \mathrm{~b}}$ and $\mathrm{p} 21^{\mathrm{Waf} / \mathrm{Cl}}$ promoters in vitro $(65,66)$. Furthermore, the suppression of Sp1 activity by miR-140 has been implicated in the maintenance of chondrocyte proliferation (67).

miRNAs in hypertrophic chondrocytes: $m i R-1, m i R-365$ and $m i R-140$. Following proliferation, chondrocytes in the center region increase in size (hypertrophic chondrocytes) and secrete and organize a different ECM (appearance of type $\mathrm{X}$ collagen). As the miRNA most repressed following chondrocyte hypertrophic differentiation, miR-1 was found to participate in chondrocytic phenotype regulation during the late stage of the differentiation process. Although the direct target remains unknown, miR-1 overexpression suppresses the expression of the major cartilaginous proteoglycan gene, aggrecan, in both human chondrocytic HCS-2/8 cells and chicken normal chondrocytes, suggesting that miR-1 participates in maintaining the integrity of the cartilage tissue (68).

By contrast, miR-365 expression is elevated in the prehypertrophic zone to stimulate chondrocyte proliferation and differentiation into hypertrophic chondrocytes (69). Of note, miR-365 is a mechano-responsive microRNA that parallels the mechanical induction of Indian hedgehog (Ihh) in primary chicken chondrocytes. Similarly, miR-365 expression coincides with the Ihh expression region in vivo. Indeed, miR-365 increases the expression of Ihh and the hypertrophic marker, type $\mathrm{X}$ collagen, whereas anti-miR-365 inhibits the expression of these genes (69). Further studies have demonstrated that histone deacetylase 4 (HDAC4), an inhibitor of chondrocyte hypertrophy (70), is an miR-365 target. miR-365 inhibits both endogenous HDAC4 protein levels and the activity of a reporter gene bearing the 3'-UTR of HDAC4 mRNA. Therefore, miR-365 inhibits HDAC4 and consequently induces the expression of its downstream molecules, Ihh and Runx2, during chondrocyte hypertrophy (69). Additionally, studies have validated experimentally that miR-140 targets HDAC4 to regulate chondrocyte differentiation, and have determined the potential role of miR-140 in long bone development (71). Furthermore, Cxcl12 was identified as a target of miR-140 by Northern blot analysis and a luciferase reporter assay (72).

miRNAs in endochondral ossification: $m i R-18 a$ and $m i R$-337. In endochondral ossification, the embryonic cartilaginous model of most bones contributes to longitudinal growth and is gradually replaced by bone. During endochondral ossification, chondrocytes proliferate, undergo hypertrophy and die. The cartilage ECM is then invaded by blood vessels, osteoclasts, bone marrow cells and osteoblasts, the last of which deposit bone on remnants of the cartilage matrix. miR-18a is the most strongly downregulated miRNA in human chondrocytic HCS-2/8 cells compared with HeLa cells, suggesting a potential role of miR-18a in chondrocytes (35). Indeed, subsequent studies have confirmed that during the late stage of chondrocyte differentiation, miR-18a targets the 3'-UTR of the CCN family protein $2 /$ connective tissue growth factor (CCN2/CTGF), a molecule important for endochondral bone formation (35). The introduction of miR-18a effectively repressed CCN2 expression in chondrocytic cells and thus significantly repressed the mature chondrocytic phenotype. The overexpression of miR-18a also repressed aggrecan and Col2al expression (35). Furthermore, many other targets, estrogen receptor, Smad2, insulin-like growth factor (IGF)-1, and hypoxia inducible factor 1- $\alpha$ (HIF1$\alpha)$, also influence the final phenotypic changes.

The expression of miR-337 is significantly downregulated and almost disappeares during the maturation phases of endochondral ossification (73). miR-337 was found to directly target the TGF- $\beta$ type II receptor (TGFBR)2, which is well known for its important roles in cartilage development (73). These data indicate that miR-18 and miRNA-337 are associated with endochondral ossification of chondrogenesis.

Other miRNAs involved in chondrogenesis: miR-23b. The expression of miR-23b is induced by $\mathrm{H}-89$, a potential mediator of chondrogenic differentiation. When upregulated by H-89, miR-23b can induce chondrogenic differentiation by negatively inhibiting the protein kinase A (PKA) signaling pathway, as evidenced by chondrogenic differentiation observed following miR-23b overexpression in human mesenchymal stem cells (hMSCs) (74).

\section{4. miRNAs in osteoarthritis}

The regulatory effects of miRNAs on OA are evident from studies comparing miRNA expression in both OA and normal 
Table II. Summary of microRNAs in OA.

\begin{tabular}{|c|c|c|c|c|}
\hline microRNAs & Targets & Regulatory role & Authors/(Refs.) & Study models \\
\hline \multirow[t]{3}{*}{$\operatorname{miR}-140$} & IGFBP-5 & OA pathogenesis $(+)$ & Tardif et al (80) & $\begin{array}{l}\text { Human cartilage obtained from femoral } \\
\text { condyles and tibial plateaus }\end{array}$ \\
\hline & ADAMTS-5 & OA pathogenesis (-) & Miyaki et al (78) & $\begin{array}{l}\text { miR-140 null mice; miR-140 transgenic (TG) mice; } \\
\text { mouse OA model; mouse antigen-induced } \\
\text { arthritis (AIA) model }\end{array}$ \\
\hline & MMP-13 & OA pathogenesis (-) & Liang et al (53) & The human cartilage cell line, C28/I2 \\
\hline miR-194 & Sox 5 & OA pathogenesis (-) & $\mathrm{Xu}$ et al (47) & $\begin{array}{l}\text { The human ASCs; } 2 \text { weeks old Wistar male rats; } \\
\text { articular chondrocytes isolated from } \\
\text { the knee joints of juvenile rats }\end{array}$ \\
\hline miR-203 & TRPV4 & OA pathogenesis (+) & Hu et al (98) & $\begin{array}{l}\text { Cartilage derived from the temporomandibular } \\
\text { joint (TMJ) of } 1 \text {-week-old female SD rats }\end{array}$ \\
\hline $\operatorname{miR}-455$ & $\begin{array}{l}\text { ACVR2B, } \operatorname{smad} 2, \\
\text { CHRDL1 }\end{array}$ & $\begin{array}{l}\text { Cartilage homeostasis; } \\
\text { OA pathogenesis }(+)\end{array}$ & Swingler et al (95) & $\begin{array}{l}\text { Human articular cartilage obtained from } \\
\text { the femoral heads of patients undergoing } \\
\text { total hip replacement surgery; SW-1353 cells; } \\
\text { C3H10T1/2 cells; 3T3 cells; ATDC5 cells }\end{array}$ \\
\hline $\operatorname{miR}-320 \mathrm{c}$ & ADAMTS-5 & OA pathogenesis (-) & Ukai et al (81) & $\begin{array}{l}\text { Cartilage tissue from patients with polydactylism, } \\
\text { anterior cruciate ligament injury, and } \\
\text { osteoarthritis undergoing total knee arthroplasty }\end{array}$ \\
\hline $\operatorname{miR}-125 b$ & ADAMTS-4 & OA pathogenesis (-) & Matsukawa et al (82) & $\begin{array}{l}\text { Cartilage tissues obtained from OA patients } \\
\text { undergoing total knee replacement }\end{array}$ \\
\hline $\operatorname{miR}-27 b$ & MMP-13 & OA pathogenesis (-) & Akhtar et al (87) & $\begin{array}{l}\text { OA cartilage samples obtained from patients } \\
\text { who underwent total joint arthroplasty; normal } \\
\text { cartilage samples obtained from trauma patients }\end{array}$ \\
\hline $\operatorname{miR}-22$ & PPAR $\alpha$, BMP7 & OA pathogenesis (-) & Iliopoulos et al (75) & $\begin{array}{l}\text { Articular cartilage obtained from femoral heads, } \\
\text { femoral condyles and tibial plateaus of patients } \\
\text { with primary osteoarthritis undergoing hip or knee } \\
\text { replacement surgery }\end{array}$ \\
\hline $\operatorname{miR}-199 a^{*}$ & $\mathrm{COX}-2$ & OA pathogenesis (-) & Akhtar et al (88) & Human chondrocytes \\
\hline miR-34a & Not detected & OA pathogenesis $(+)$ & Abouheif et al (61) & Rat osteoarthritis model \\
\hline $\operatorname{miR}-181 b$ & Not detected & OA pathogenesis $(+)$ & Song et al (94) & $\begin{array}{l}\text { Mesenchymal cells derived from the distal tips } \\
\text { of Hamburger-Hamilton }(\mathrm{HH}) \text { stage } 22 / 23 \text { embryo } \\
\text { leg buds of fertilized white Leghorn chicken } \\
\text { eggs; human chondrocytes }\end{array}$ \\
\hline miR-146 & Not detected & OA pathogenesis (-) & Jones et al (91) & $\begin{array}{l}\text { Cartilage and bone obtained from patients } \\
\text { with OA or not }\end{array}$ \\
\hline $\begin{array}{l}\operatorname{miR}-193 b \\
\operatorname{miR}-199 a-3 p\end{array}$ & $\begin{array}{l}\text { Aggrecan, type } 2 \\
\text { collagen, Sox } 9\end{array}$ & OA pathogenesis $(+)$ & Ukai et al (81) & $\begin{array}{l}\text { Cartilage tissue from patients with polydactylism, } \\
\text { anterior cruciate ligament injury, and osteoarthritis } \\
\text { undergoing total knee arthroplasty }\end{array}$ \\
\hline miR-675 & Not detected & Cartilage homeostasis $(+)$ & Dudek et al (96) & Human articular cartilage \\
\hline
\end{tabular}

OA, osteoarthritis; MMP, matrix metalloproteinase; BMP7, bone morphogenetic protein 7; COX-2, cyclooxygenase-2.

articular tissues. Iliopoulos et al (75) measured the expression of 365 miRNAs and identified 9 significantly upregulated miRNAs and 7 downregulated miRNAs in OA cartilage, compared with normal controls, suggesting that miRNAs are involved in OA development. In this review, we summarize the role of miRNAs in the development of OA (Table II and Fig. 3).

miRNAs regulate proteolytic enzymes in OA: miR-140, $m i R-320 c$, $m i R-125 b$ and $m i R-27 b$. ADAMTS-5 is a critical proteolytic enzyme causing aggrecan degradation in cartilage. ADAMTS-5-deficient animals are resistant to cartilage degeneration in the surgical OA model and inflammatory arthritis model (76,77). miR-140 is an miRNA that may regulate ADAMTS-5 expression. miR-140 is specifically expressed in the cartilage tissue of mouse embryos during long and flat bone development, and is required for cartilage homeostasis and skeletal development (71). miR-140 expression is decreased in OA chondrocytes following IL-1 $\beta$ stimulation (55). Similarly, 


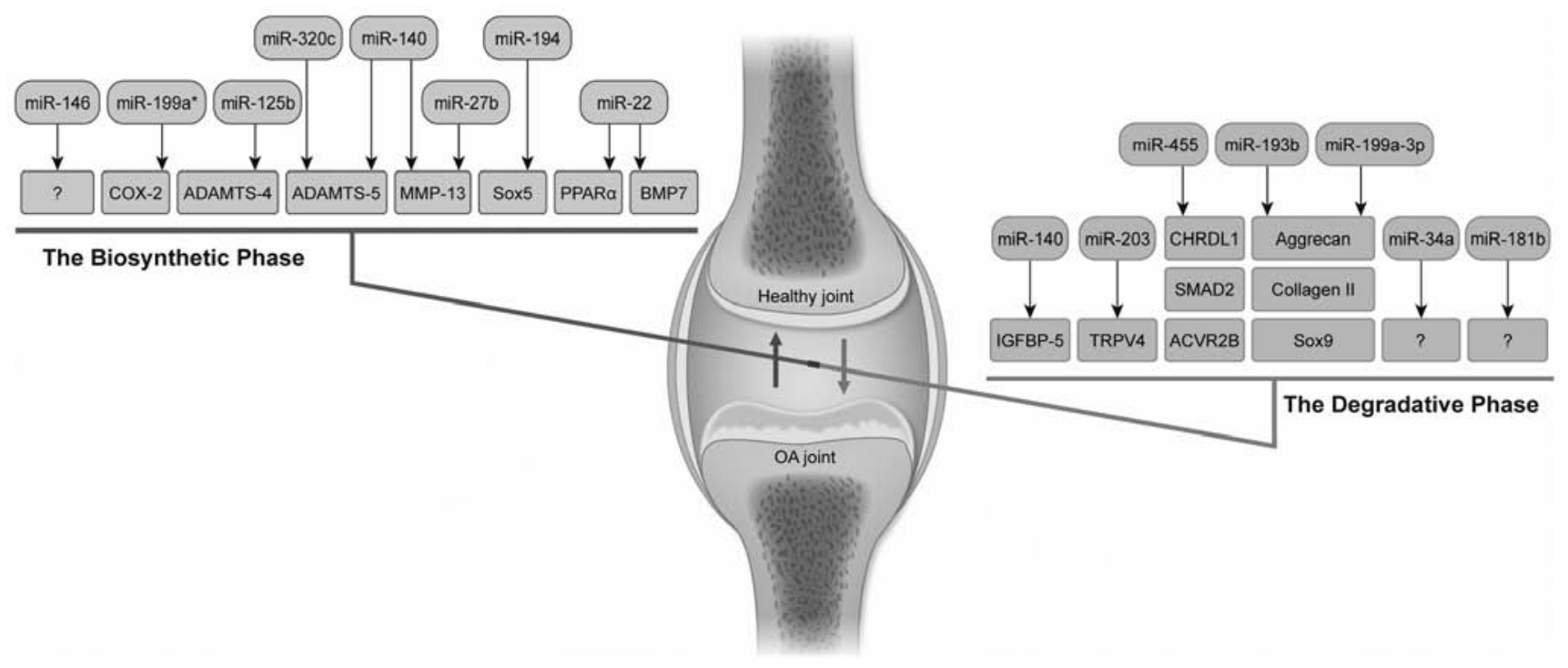

Figure 3. Key microRNAs (miRNAs) and factors balancing the anabolic and catabolic pathways of the osteoarthritis (OA) joint. Anabolic mechanisms are presented on the left side of the figure and catabolic pathways are presented on the right side of the figure.

mice lacking miR-140 are more prone to developing severe OA than are wild-type mice. miR-140 overexpression in cartilage also protects mice from cartilage degradation (78). Furthermore, miR-140 expression is decreased in the knee synovial fluid in patients with OA, and is negatively related to the severity of OA. This may be related to OA occurrence and development, and may therefore represent a potential molecular target for early diagnosis (79). It has also been revealed that miR-140 suppresses ADAMTS-5 expression, thereby inhibiting OA-like changes (78). Additionally, miR-140 was identified as a negative feedback regulator of MMP-13, a wellcharacterized key player in cartilage biology and OA pathology owing to its capacity to degrade collagens and numerous other matrix components (53). Furthermore, IGFBP-5, a direct target of miR-140, is expressed in human chondrocytes with its level being significantly lower in OA (80).

Additionally, miR-320c was identified in the regulation of ADAMTS-5 expression, although miR-320c expression is apparently downregulated with age in human cartilage tissue. Of note, ADAMTS-5 expression was downregulated in the miR-320c mimic group and upregulated in the inhibitor group (81), suggesting that miR-320c is involved in OA by regulating ADAMTS-5.

miR-125b expression is significantly lower in OA chondrocytes than in normal chondrocytes. miR-125b seeds sequence in 3'-UTR of human aggrecanase-1 (ADAMTS-4) mRNA, and suppresses ADAMTS-4 mRNA expression by $72 \%$ and protein production by $62 \%$ following IL-1 $\beta$ stimulation (82). Increased ADAMTS-4 expression is an important factor in OA and other joint diseases, and therefore, it is important for the control of miR-125b expression, which may represent a novel approach for OA prevention and treatment. Additionally, in other cell types $(83,84)$, miR-125b suppresses MMP-13, an important collagenase in articular cartilage (85), and vascular endothelialc adherin, a regulator of angiogenesis, whose levels are increased in OA-affected joints (86).
The human genome contains 2 miR-27 genes (miR-27a and miR-27b) on chromosomes 19 and 9, respectively. Their mature products differ by only 1 nucleotide in the $3^{\prime}$ region. Of note, no change in miR-27a expression in OA has been reported. By contrast, miR-27b was identified as a downregulated miRNA in IL-1 $\beta$-stimulated human OA chondrocytes. By using the 3'-UTR reporter assay and gain- or loss-of-function experiments, MMP-13 was identified as a direct target of miR-27b. MMP-13 exhibits broad substrate specificity and can cleave types I, II, III, IV, X and XIV collagen; aggrecan; and fibronectin, with the highest activity being directed toward type II collagen in OA. Thus, upregulating miR-27b expression or preventing its downregulation in vivo may present a novel therapeutic and/or preventive approach in OA treatment (87). Thus, several miRNAs, including miR-140, miR-320c, miR-125b and $\mathrm{miR}-27 \mathrm{~b}$, participate OA pathogenesis by targeting the proteolytic enzymes.

miRNAs are involved in both chondrogenesis and OA: miR-194, $m i R-199 a^{*}$ and $m i R-34 a$. As discussed above, several miRNAs play important roles in regulating chondrogenesis. In addition to their physiological roles, these miRNAs appear to participate in OA pathogenesis. For instance, the miR-194 level has been shown to be reduced during chondrogenic differentiation by directly targeting Sox 5 expression. By contrast, miR-194 expression was upregulated and Sox 5 expression was downregulated in OA (47). Additionally, miR-199a* targeted not only Sox 9 in chondrocytes but also cyclooxygenase-2 (COX-2) in OA chondrocytes (88). miR-199a* directly suppressed the luciferase activity of a COX-2 3'-UTR reporter construct and inhibited IL- $1 \beta$-induced expression of COX- 2 in OA chondrocytes. The modulation of miR-199a* expression also significantly inhibited the IL-1 $\beta$-induced upregulation of $\mathrm{mPGES} 1$ and prostaglandin E2 production in OA chondrocytes. The activation of p38-MAPK downregulated miR-199a* expression and induced COX-2 expression (88). Collectively, these data suggest that 
miR-199a* participates in OA development, and thus represents another potential target for OA treatment.

Additionally, miR-34a is a negative modulator of chondrogenesis, particularly in chondroblast migration, by targeting EphA5, which inhibits cellular condensation. Interestingly, miRNA-34a silencing can prevent chondrocyte apoptosis, suggesting the involvement of miR-34a in cell apoptosis $(61,89,90)$. In agreement with these findings, miR-34a expression was also upregulated in human OA cartilage (91). Together, these data suggest that miR-34a participates in promoting chondrocyte apoptosis during OA development.

\section{Other miRNAs in $O A$}

Downregulated miRNAs in OA: miR-146. miR-146 expression is high in low-grade OA cartilage and inversely correlates with cartilage degeneration in late-stage OA cartilage $(91,92)$. Thus, the miR-146 expression pattern appears to depend on OA severity. A target of miR-146 in chondrocytes has not been detected yet; however, TNF- $\alpha$ production is significantly reduced by miR-146 overexpression, indicating that miR-146 participates in suppressing inflammation in OA (91). This was further evidenced by the finding that miR-146a controls knee-joint homeostasis and OA-associated algesia by balancing inflammatory responses in cartilage and synovium (93). Hence, miR-146a may target inflammation and thus be useful for the treatment of both cartilage degeneration and pain symptoms in OA.

Upregulated miRNAs in OA: miR-193b, miR-199a-3p, miR-181b, miR-22, miR-455 and miR-675. miR-193b and miR-199a-3p expression are upregulated with age in human cartilage tissue, and these may be involved in chondrocyte aging by regulating aggrecan, type 2 collagen and Sox 9 (81). Therefore, miR-199a-3p and miR-193b are involved in the senescence of chondrocytes with currently unknown targets.

miR-181b expression is significantly downregulated during chondrogenic differentiation of TGF- $\beta 3$-stimulated limb mesenchymal cells, but is significantly upregulated in OA chondrocytes (94). Although a direct target has not identified, miR-181b attenuation reduced MMP-13 expression, while inducing type II collagen expression. Furthermore, anti-miR-181b overexpression significantly reduced cartilage destruction in mice (94). These data suggest that miR-181b is a negative regulator of cartilage development, and miR-181b inhibition may be an effective therapeutic strategy for cartilagerelated diseases.

miR-22 is another miRNA upregulated in human OA cartilage. miR-22 can directly downregulate BMP7 at the mRNA level and PPAR $\alpha$ at the protein level, which subsequently upregulates IL-1 $\beta$ and MMP-13 expression in chondrocytes, which is detrimental to the cartilage ECM. As previously demonstrated, miR-22 inhibition in osteoarthritic chondrocytes upregulates PPAR $\alpha$ (4.9-fold) and BMP7 (5.8-fold) expression, blocks the inflammatory process through the inhibition of IL1B (7.6-fold), inhibits catabolic changes, such as MMP-13 expression (7.9-fold) and activates the cartilage repair protein aggrecan (3.1-fold), suggesting the therapeutic potential of miRNA inhibition in OA (75).

miR-455 resides within an intron of Col27al that encodes a cartilage collagen. When human OA cartilage was compared with cartilage obtained from patients with femoral neck fractures, miR-140-5p expression increased in OA cartilage.
In situ hybridization revealed miR-455-3p expression in the developing limbs of chicks and mice and in human OA cartilage (95). ACVR2B, Smad2 and CHRDL1 are direct targets of miR-455-3p, and these may mediate its functional impact on TGF signaling. miR-455 is expressed during chondrogenesis and in adult articular cartilage, where it can regulate TGF signaling, suppressing the Smad2/3 pathway. Diminished signaling through this pathway during the aging process and in OA chondrocytes contributes to cartilage destruction. The increased miR-455 expression in OA may exacerbate this process and may contribute to the pathogenesis of the disease (95).

miR-675 is encoded by lncRNA H19, which is highly expressed as the most abundant cartilage matrix genes Col2a1 and aggrecan (96). Both miR-675 and lncRNA H19 are highly expressed in articular cartilage and are upregulated in $\mathrm{OA}$ cartilage. Stress-induced regulation of H19 expression by hypoxic signaling and inflammation suggests that lncRNA H19 acts as a metabolic correlate in cartilage (97). Though a direct target gene remains to be identified, the H19-encoded miR-675 may modulate collagen type II levels via an unrecognized molecule (97).

miR-203: Transient receptor potential vanilloid 4 (TRPV4) is a member of TRP superfamily of $\mathrm{Ca}^{2+}$-permeable non-selective cation channels. miR-203 can upregulate nitric oxide (NO) expression in female rat mandibular condylar chondrocytes by targeting TRPV4. NO is a critical mediator of the disrupted processes implicated in OA pathophysiology (98).

\section{Conclusion}

Undoubtedly, miRNAs, which play a critical role in one-third of human transcriptional regulation, represent a novel breakthrough in the field of genetic engineering. Over the past few years, remarkable progress has been made in the study of miRNAs, as an increasing number of tissue-specific miRNAs have been identified in chondrocytes. In conclusion, miRNAs appear to have significant potential for OA diagnostics and therapeutics and cartilage tissue engineering. However, their potential will only be realized through further research to identify potential miRNA target sites and the associated mechanisms.

\section{Acknowledgements}

This study was supported by a grant from the National Natural Science Foundation of China (no.81171729), a scientific research grant from the National Natural Science Foundation for the Youth of China (Grant No. 81201364), a scientific research grant for the youth of Shanghai (Grant No. ZZjdyx 2097), a scientific research grant from the 985 project - Stem Cell and Regenerative Medicine Centre, an innovative research grant from the Shanghai Municipal Education Commission (Grant No. 13YZ031) and a fund for Key Disciplines of Shanghai Municipal Education Commission (J50206).

\section{References}

1. Iannone F and Lapadula G: The pathophysiology of osteoarthritis. Aging Clin Exp Res 15: 364-372, 2003. 
2. Mortellaro CM: Pathophysiology of osteoarthritis. Vet Res Commun 27 (Suppl 1): S75-S78, 2003.

3. Martel-Pelletier J: Pathophysiology of osteoarthritis. Osteoarthritis Cartilage 12 (Suppl A): S31-S33, 2004.

4. Mandelbaum B and Waddell D: Etiology and pathophysiology of osteoarthritis. Orthopedics 28 (Suppl 2): s207-s214, 2005.

5. Felson DT, Lawrence RC, Dieppe PA, et al: Osteoarthritis: new insights. Part 1: the disease and its risk factors. Ann Intern Med 133: 635-646, 2000.

6. Lawrence RC, Felson DT, Helmick CG, et al: Estimates of the prevalence of arthritis and other rheumatic conditions in the United States. Part II. Arthritis Rheum 58: 26-35, 2008

7. Naumann A, Dennis JE, Awadallah A, et al: Immunochemical and mechanical characterization of cartilage subtypes in rabbit. J Histochem Cytochem 50: 1049-1058, 2002.

8. Wong $\mathrm{M}$ and Carter DR: Articular cartilage functional histomorphology and mechanobiology: a research perspective. Bone 33 $1-13,2003$.

9. Burgeson RE, Hebda PA, Morris NP and Hollister DW: Human cartilage collagens. Comparison of cartilage collagens with human type V collagen. J Biol Chem 257: 7852-7856, 1982.

10. Eyre D: Collagen of articular cartilage. Arthritis Res 4: 30-35, 2002.

11. Poole AR, Kojima T, Yasuda T, Mwale F, Kobayashi M and Laverty S: Composition and structure of articular cartilage: a template for tissue repair. Clin Orthop Relat Res 391: S26-S33, 2001.

12. Knudson CB and Knudson W: Cartilage proteoglycans. Semin Cell Dev Biol 12: 69-78, 2001

13. Cawston TE and Wilson AJ: Understanding the role of tissue degrading enzymes and their inhibitors in development and disease. Best Pract Res Clin Rheumatol 20: 983-1002, 2006.

14. Plaas A, Osborn B, Yoshihara Y, et al: Aggrecanolysis in human osteoarthritis: confocal localization and biochemical characterization of ADAMTS5-hyaluronan complexes in articular cartilages. Osteoarthritis Cartilage 15: 719-734, 2007.

15. Wu W, Billinghurst RC, Pidoux I, et al: Sites of collagenase cleavage and denaturation of type II collagen in aging and osteoarthritic articular cartilage and their relationship to the distribution of matrix metalloproteinase 1 and matrix metalloproteinase 13. Arthritis Rheum 46: 2087-2094, 2002.

16. Bartel DP: MicroRNAs: genomics, biogenesis, mechanism, and function. Cell 116: 281-297, 2004.

17. Cordes KR and Srivastava D: MicroRNA regulation of cardiovascular development. Circ Res 104: 724-732, 2009.

18. Fabian MR, Sonenberg N and Filipowicz W: Regulation of mRNA translation and stability by microRNAs. Annu Rev Biochem 79: 351-379, 2010.

19. Liu N and Olson EN: MicroRNA regulatory networks in cardiovascular development. Dev Cell 18: 510-525, 2010.

20. Wang DZ: MicroRNAs in cardiac development and remodeling. Pediatr Cardiol 31: 357-362, 2010

21. Zhao Y and Srivastava D: A developmental view of microRNA function. Trends Biochem Sci 32: 189-197, 2007

22. Farh KK, Grimson A, Jan C, et al: The widespread impact of mammalian MicroRNAs on mRNA repression and evolution. Science 310: 1817-1821, 2005.

23. Calin GA and Croce CM: MicroRNA signatures in human cancers. Nat Rev Cancer 6: 857-866, 2006.

24. Chendrimada TP, Gregory RI, Kumaraswamy E, et al: TRBP recruits the Dicer complex to Ago2 for microRNA processing and gene silencing. Nature 436: 740-744, 2005

25. Lee Y, Ahn C, Han J, et al: The nuclear RNase III Drosha initiates microRNA processing. Nature 425: 415-419, 2003.

26. Gregory RI, Yan KP, Amuthan G, et al: The Microprocessor complex mediates the genesis of microRNAs. Nature 432: 235-240, 2004

27. Denli AM, Tops BB, Plasterk RH, Ketting RF and Hannon GJ: Processing of primary microRNAs by the Microprocessor complex. Nature 432: 231-235, 2004

28. Small EM and Olson EN: Pervasive roles of microRNAs in cardiovascular biology. Nature 469: 336-342, 2011.

29. Ghayor C, Chadjichristos C, Herrouin JF, et al: Sp3 represses the Sp1-mediated transactivation of the human COL2A1 gene in primary and de-differentiated chondrocytes. J Biol Chem 276: 36881-36895, 2001

30. Zhang Z, Kang Y, Zhang H, et al: Expression of microRNAs during chondrogenesis of human adipose-derived stem cells. Osteoarthritis Cartilage 20: 1638-1646, 2012.

31. Cancedda R, Descalzi Cancedda F and Castagnola P: Chondrocyte differentiation. Int Rev Cytol 159: 265-358, 1995.
32. Yang B, Guo H, Zhang Y, Chen L, Ying D and Dong $S$ : MicroRNA-145 regulates chondrogenic differentiation of mesenchymal stem cells by targeting Sox9. PLoS One 6: e21679, 2011.

33. Martinez-Sanchez A, Dudek KA and Murphy CL: Regulation of human chondrocyte function through direct inhibition of cartilage master regulator SOX9 by microRNA-145 (miRNA-145). J Biol Chem 287: 916-924, 2012

34. Ning G, Liu X, Dai M, Meng A and Wang Q: MicroRNA-92a upholds Bmp signaling by targeting noggin 3 during pharyngeal cartilage formation. Dev Cell 24: 283-295, 2013.

35. Ohgawara T, Kubota S, Kawaki H, et al: Regulation of chondrocytic phenotype by micro RNA 18a: involvement of Ccn2/Ctgf as a major target gene. FEBS Lett 583: 1006-1010, 2009.

36. Soullier S, Jay P, Poulat F, Vanacker JM, Berta P and Laudet V: Diversification pattern of the HMG and SOX family members during evolution. J Mol Evol 48: 517-527, 1999.

37. Wright E, Hargrave MR, Christiansen J, et al: The Sry-related gene Sox 9 is expressed during chondrogenesis in mouse embryos. Nat Genet 9: 15-20, 1995.

38. Bi W, Deng JM, Zhang Z, Behringer RR and de Crombrugghe B: Sox9 is required for cartilage formation. Nat Genet 22: 85-89, 1999.

39. Ikeda T, Kawaguchi H, Kamekura S, et al: Distinct roles of Sox 5 , Sox6, and Sox9 in different stages of chondrogenic differentiation. J Bone Miner Metab 23: 337-340, 2005.

40. Bell DM, Leung KK, Wheatley SC, et al: SOX9 directly regulates the type-II collagen gene. Nat Genet 16: 174-178, 1997.

41. Zhang P, Jimenez SA and Stokes DG: Regulation of human COL9A1 gene expression. Activation of the proximal promoter region by SOX9. J Biol Chem 278: 117-123, 2003.

42. Liu Y, Li H, Tanaka K, Tsumaki N and Yamada Y: Identification of an enhancer sequence within the first intron required for cartilage-specific transcription of the alpha2(XI) collagen gene. J Biol Chem 275: 12712-12718, 2000.

43. Sekiya I, Tsuji K, Koopman P, et al: SOX9 enhances aggrecan gene promoter/enhancer activity and is up-regulated by retinoic acid in a cartilage-derived cell line, TC6. J Biol Chem 275: 10738-10744, 2000.

44. Tew SR, Li Y, Pothacharoen P, Tweats LM, Hawkins RE and Hardingham TE: Retroviral transduction with SOX9 enhances re-expression of the chondrocyte phenotype in passaged osteoarthritic human articular chondrocytes. Osteoarthritis Cartilage 13: 80-89, 2005.

45. Cucchiarini M, Thurn T, Weimer A, Kohn D, Terwilliger EF and Madry H: Restoration of the extracellular matrix in human osteoarthritic articular cartilage by overexpression of the transcription factor SOX9. Arthritis Rheum 56: 158-167, 2007.

46. Dai L, Zhang X, Hu X, Zhou C and Ao Y: Silencing of microRNA-101 prevents IL-1beta-induced extracellular matrix degradation in chondrocytes. Arthritis Res Ther 14: R268, 2012.

47. Xu J, Kang Y, Liao WM and Yu L: MiR-194 regulates chondrogenic differentiation of human adipose-derived stem cells by targeting Sox5. PLoS One 7: e31861, 2012.

48. Parvizi J, Zmistowski B, Berbari EF, et al: New definition for periprosthetic joint infection: from the Workgroup of the Musculoskeletal Infection Society. Clin Orthop Relat Res 469: 2992-2994, 2011.

49. Hatakeyama Y, Nguyen J, Wang X, Nuckolls GH and Shum L: Smad signaling in mesenchymal and chondroprogenitor cells. J Bone Joint Surg Am 85-A (Suppl 3): S13-S18, 2003.

50. Pan Q, Yu Y, Chen Q, et al: Sox9, a key transcription factor of bone morphogenetic protein-2-induced chondrogenesis, is activated through BMP pathway and a CCAAT box in the proximal promoter. J Cell Physiol 217: 228-241, 2008.

51. Denker AE, Nicoll SB and Tuan RS: Formation of cartilagelike spheroids by micromass cultures of murine C3H10T1/2 cells upon treatment with transforming growth factor-beta 1. Differentiation 59: 25-34, 1995.

52. Lin EA, Kong L, Bai XH, Luan Y and Liu CJ: miR-199a, a bone morphogenic protein 2-responsive MicroRNA, regulates chondrogenesis via direct targeting to Smad1. J Biol Chem 284: $11326-11335,2009$

53. Liang ZJ, Zhuang H, Wang GX, et al: MiRNA-140 is a negative feedback regulator of MMP-13 in IL-1beta-stimulated human articular chondrocyte C28/I2 cells. Inflamm Res 61: 503-509, 2012.

54. Buechli ME, Lamarre J and Koch TG: MicroRNA-140 expression during chondrogenic differentiation of equine cord blood-derived mesenchymal stromal cells. Stem Cells Dev 22: 1288-1296, 2013 
55. Miyaki S, Nakasa T, Otsuki S, et al: MicroRNA-140 is expressed in differentiated human articular chondrocytes and modulates interleukin-1 responses. Arthritis Rheum 60: 2723-2730, 2009.

56. Nicolas FE, Pais H, Schwach F, et al: mRNA expression profiling reveals conserved and non-conserved miR-140 targets. RNA Biol 8: 607-615, 2011

57. Pais H, Nicolas FE, Soond SM, et al: Analyzing mRNA expression identifies Smad3 as a microRNA-140 target regulated only at protein level. RNA 16: 489-494, 2010

58. Nakamura $Y$, Inloes $J B$, Katagiri $T$ and Kobayashi $T$ : Chondrocyte-specific microRNA-140 regulates endochondral bone development and targets Dnpep to modulate bone morphogenetic protein signaling. Mol Cell Biol 31: 3019-3028, 2011.

59. Song J, Kim D and Jin EJ: MicroRNA-488 suppresses cell migration through modulation of the focal adhesion activity during chondrogenic differentiation of chick limb mesenchymal cells. Cell Biol Int 35: 179-185, 2011.

60. Kim D, Song J, Kim S, Chun CH and Jin EJ: MicroRNA-34a regulates migration of chondroblast and IL-1beta-induced degeneration of chondrocytes by targeting EphA5. Biochem Biophys Res Commun 415: 551-557, 2011.

61. Abouheif MM, Nakasa T, Shibuya H, Niimoto T, Kongcharoensombat W and Ochi M: Silencing microRNA-34a inhibits chondrocyte apoptosis in a rat osteoarthritis model in vitro. Rheumatology (Oxford) 49: 2054-2060, 2010.

62. Kim D, Song J and Jin EJ: MicroRNA-221 regulates chondrogenic differentiation through promoting proteosomal degradation of slug by targeting Mdm2. J Biol Chem 285: 26900-26907, 2010.

63. Dunn W, DuRaine G and Reddi AH: Profiling microRNA expression in bovine articular cartilage and implications for mechanotransduction. Arthritis Rheum 60: 2333-2339, 2009.

64. Magee C, Nurminskaya M, Faverman L, Galera P and Linsenmayer TF: SP3/SP1 transcription activity regulates specific expression of collagen type $\mathrm{X}$ in hypertrophic chondrocytes. J Biol Chem 280: 25331-25338, 2005.

65. Kavurma MM and Khachigian LM: Sp1 inhibits proliferation and induces apoptosis in vascular smooth muscle cells by repressing p21WAF1/Cip1 transcription and cyclin D1-Cdk4-p21WAF1/ Cip1 complex formation. J Biol Chem 278: 32537-32543, 2003.

66. Deniaud E, Baguet J, Chalard R, et al: Overexpression of transcription factor $\mathrm{Sp} 1$ leads to gene expression perturbations and cell cycle inhibition. PLoS One 4: e7035, 2009.

67. Yang J, Qin S, Yi C, et al: MiR-140 is co-expressed with Wwp2-C transcript and activated by Sox 9 to target Sp1 in maintaining the chondrocyte proliferation. FEBS Lett 585: 2992-2997, 2011.

68. Sumiyoshi K, Kubota S, Ohgawara T, et al: Identification of miR-1 as a micro RNA that supports late-stage differentiation of growth cartilage cells. Biochem Biophys Res Commun 402: 286-290, 2010

69. Guan YJ, Yang X, Wei L and Chen Q: MiR-365: a mechanosensitive microRNA stimulates chondrocyte differentiation through targeting histone deacetylase 4. FASEB J 25: 4457-4466, 2011.

70. Vega RB, Matsuda K, Oh J, et al: Histone deacetylase 4 controls chondrocyte hypertrophy during skeletogenesis. Cell 119: 555-566, 2004.

71. Tuddenham L, Wheeler G, Ntounia-Fousara S, et al: The cartilage specific microRNA-140 targets histone deacetylase 4 in mouse cells. FEBS Lett 580: 4214-4217, 2006.

72. Nicolas FE, Pais H, Schwach F, et al: Experimental identification of microRNA-140 targets by silencing and overexpressing miR-140. RNA 14: 2513-2520, 2008.

73. Zhong N, Sun J, Min Z, et al: MicroRNA-337 is associated with chondrogenesis through regulating TGFBR 2 expression. Osteoarthritis Cartilage 20: 593-602, 2012.

74. Ham O, Song BW, Lee SY, et al: The role of microRNA-23b in the differentiation of MSC into chondrocyte by targeting protein kinase A signaling. Biomaterials 33: 4500-4507, 2012.

75. Iliopoulos D, Malizos KN, Oikonomou P and Tsezou A: Integrative microRNA and proteomic approaches identify novel osteoarthritis genes and their collaborative metabolic and inflammatory networks. PLoS One 3: e3740, 2008.

76. Glasson SS, Askew R, Sheppard B, et al: Deletion of active ADAMTS5 prevents cartilage degradation in a murine model of osteoarthritis. Nature 434: 644-648, 2005.

77. Stanton H, Rogerson FM, East CJ, et al: ADAMTS5 is the major aggrecanase in mouse cartilage in vivo and in vitro. Nature 434 648-652, 2005 .
78. Miyaki S, Sato T, Inoue A, et al: MicroRNA-140 plays dual roles in both cartilage development and homeostasis. Genes Dev 24: $1173-1185,2010$

79. Zhang M, Liu L, Xiao T and Guo W: Detection of the expression level of miR-140 using realtime fluorescent quantitative PCR in knee synovial fluid of osteoarthritis patients. Zhong Nan Da Xue Xue Bao Yi Xue Ban 37: 1210-1214, 2012 (In Chinese).

80. Tardif G, Hum D, Pelletier JP, Duval N and Martel-Pelletier J: Regulation of the IGFBP-5 and MMP-13 genes by the microRNAs miR-140 and miR-27a in human osteoarthritic chondrocytes. BMC Musculoskelet Disord 10: 148, 2009.

81. Ukai T, Sato M, Akutsu H, Umezawa A and Mochida J: MicroRNA-199a-3p, microRNA-193b, and microRNA-320c are correlated to aging and regulate human cartilage metabolism. J Orthop Res 30: 1915-1922, 2012

82. Matsukawa T, Sakai T, Yonezawa T, et al: MicroRNA-125b regulates the expression of aggrecanase-1 (ADAMTS-4) in human osteoarthritic chondrocytes. Arthritis Res Ther 15: R28, 2013.

83. Xu N, Zhang L, Meisgen F, et al: MicroRNA-125b down-regulates matrix metallopeptidase 13 and inhibits cutaneous squamous cell carcinoma cell proliferation, migration, and invasion. J Biol Chem 287: 29899-29908, 2012.

84. Muramatsu F, Kidoya H, Naito H, Sakimoto S and Takakura N: microRNA-125b inhibits tube formation of blood vessels through translational suppression of VE-cadherin. Oncogene 32: 414-421, 2013.

85. Little CB, Barai A, Burkhardt D, et al: Matrix metalloproteinase 13-deficient mice are resistant to osteoarthritic cartilage erosion but not chondrocyte hypertrophy or osteophyte development. Arthritis Rheum 60: 3723-3733, 2009.

86. Mapp PI and Walsh DA: Mechanisms and targets of angiogenesis and nerve growth in osteoarthritis. Nat Rev Rheumatol 8: 390-398, 2012.

87. Akhtar N, Rasheed Z, Ramamurthy S, Anbazhagan AN, Voss FR and Haqqi TM: MicroRNA-27b regulates the expression of matrix metalloproteinase 13 in human osteoarthritis chondrocytes. Arthritis Rheum 62: 1361-1371, 2010.

88. Akhtar N and Haqqi TM: MicroRNA-199a* regulates the expression of cyclooxygenase-2 in human chondrocytes. Ann Rheum Dis 71: 1073-1080, 2012.

89. He L, He X, Lim LP, et al: A microRNA component of the p53 tumour suppressor network. Nature 447: 1130-1134, 2007.

90. Chang TC, Wentzel EA, Kent OA, et al: Transactivation of miR-34a by 53 broadly influences gene expression and promotes apoptosis. Mol Cell 26: 745-752, 2007.

91. Jones SW, Watkins G, Le Good N, et al: The identification of differentially expressed microRNA in osteoarthritic tissue that modulate the production of TNF-alpha and MMP13. Osteoarthritis Cartilage 17: 464-472, 2009.

92. Yamasaki K, Nakasa T, Miyaki S, et al: Expression of MicroRNA-146a in osteoarthritis cartilage. Arthritis Rheum 60: 1035-1041, 2009

93. Li X, Gibson G, Kim JS, et al: MicroRNA-146a is linked to pain-related pathophysiology of osteoarthritis. Gene 480: 34-41, 2011.

94. Song J, Lee M, Kim D, Han J, Chun CH and Jin EJ: MicroRNA-181b regulates articular chondrocytes differentiation and cartilage integrity. Biochem Biophys Res Commun 431: 210-214, 2013.

95. Swingler TE, Wheeler G, Carmont V, et al: The expression and function of microRNAs in chondrogenesis and osteoarthritis. Arthritis Rheum 64: 1909-1919, 2012.

96. Dudek KA, Lafont JE, Martinez-Sanchez A and Murphy CL: Type II collagen expression is regulated by tissue-specific miR-675 in human articular chondrocytes. J Biol Chem 285: 24381-24387, 2010

97. Steck E, Boeuf S, Gabler J, et al: Regulation of H19 and its encoded microRNA-675 in osteoarthritis and under anabolic and catabolic in vitro conditions. J Mol Med (Berl) 90: 1185-1195, 2012.

98. Hu F, Zhu W and Wang L: MicroRNA-203 up-regulates nitric oxide expression in temporomandibular joint chondrocytes via targeting TRPV4. Arch Oral Biol: Nov 16, 2012 (Epub ahead of print). doi: 10.1016/j.archoralbio.2012.08.013 